\title{
Evidence of Antibiotic Resistance from Population-Based Studies: A Narrative Review
}

This article was published in the following Dove Press journal:

Infection and Drug Resistance

\section{Elisa Giacomini \\ Valentina Perrone \\ Davide Alessandrini \\ Daniela Paoli \\ Carmela Nappi \\ Luca Degli Esposti}

CliCon S.r.l. Health, Economics \&

Outcomes Research, Ravenna, Italy
Correspondence: Valentina Perrone Clicon S.r.l, Health, Economics and Outcomes Research, Via Salara, 36, Ravenna, 48100, Italy

Tel +3954438393

$\mathrm{Fax}+39544212699$

Email valentina.perrone@clicon.it
Abstract: The 20th century witnessed the dawn of the antibiotic revolution and is now facing the rising phenomenon of antibiotic resistance. In this narrative review, we aim to describe antibiotic resistance in clinical practice settings through population-based studies from different countries reporting the role of misuse of antibiotics in the development of resistance and the clinical and economic burden associated. The misuse of antibiotics was documented in the wide population as well as in hospitals and care facilities. It was mainly reported as over-use and inappropriate prescribing. Improper dosage regimens and longer treatment duration were regarded as pivotal factors related to antibiotic resistance; the emerging strategy of "antibiotic-de-escalation" could be the key to overcome these issues. The investigation of the self-medication attitude revealed widespread antibiotic use without following medical instructions or medical consultation. Moreover, several studies established the association of antibiotic resistance with increased risk of longer hospitalizations and mortality, highlighting the heavy clinical and economic burden of this phenomenon. In this narrative review, the widespread inappropriate use of antibiotics emerged as one of the main causes of antibiotic resistance, which negative outcomes call for the development of antibiotic stewardship programs and global surveillance networks.

Keywords: antibiotic utilization, antimicrobial resistance, real-world

\section{Introduction}

\section{Overview of Antibiotic Resistance}

The discovery of penicillin by Alexander Fleming in 1928 marked the beginning of the antibiotics era, it looked like the struggle of mankind against infections was finally over thanks to these powerful weapons. Use of antibiotics and the introduction of vaccination have increased life expectancy, decreased childhood mortality and allowed invasive surgery and chemotherapy treatments. ${ }^{1}$ However, we are still far away to win that battle, as we have to face the threat of antibiotic resistance (ABR).

ABR is defined as the ability of microorganisms to survive to the exposure of antibiotics that normally would be able to kill them or to stop their growth. ${ }^{2}$ The rising of a phenotype resistant to antimicrobial agents depends on several factors such as degree of resistance expression of the microorganism or its capability to tolerate resistance mechanism, to cite a few. ${ }^{3}$ Bacteria may have intrinsic resistance or acquire resistance from either mutations in cell genes (chromosomal mutation) leading to cross-resistance, or gene transfer from one microorganism to another mediated by plasmids, transposons, integrons and bacteriophages. When resistance determinants 
are on plasmids, resistant microorganisms will spread quickly. ${ }^{3}$ Several biochemical types of resistance mechanisms can be used by the bacteria to protect themselves from various agents, the most important mechanisms being enzymatic degradation, target alteration, decreased uptake and overexpression of efflux pump proteins. ${ }^{4}$

Even though this phenomenon is a natural process observed in clinical practice since the first-generation antibiotics, ${ }^{5}$ ABR is nowadays regarded as a global public health concern due to the rising rate of resistance development and spreading and the lack of new drugs able to contain it. ${ }^{6}$ The development of non-susceptibility to the novel antibiotics is deemed as inevitable and potentially leads to a shortened period of clinical usefulness of these drugs. ${ }^{7}$ Based on their resistance levels and clinical significance, the World Health Organization declared the so-called "ESKAPE" pathogens (E: Enterococcus faecium, S: Staphylococcus aureus or Stenotrophomonas maltophilia, K: Klebsiella pneumoniae or C: Clostridioides difficile, A: Acinetobacter baumannii, P: Pseudomonas aeruginosa, E: Enterobacteriaceae) as priority pathogens for pharmaceutical companies. ${ }^{7}$

The dimension of ABR is also mirrored in the increasing number of publications focused on this topic over the past seventy years (Figure 1).

The Sixty-eight World Health Assembly responded to the rising alarm of ABR by launching in 2015 a global action plan with the aim to optimize the use of antibiotic agents, to make these drugs accessible to all who need them and to increase knowledge and awareness on ABR, thus ensuring the continuity of successful treatment and prevention of infectious diseases. ${ }^{8}$

ABR affects all areas of health, from human sector to animal and environment, thus involving society as a whole. ${ }^{8}$ Antibiotics are indeed prescribed not only for the treatment of infectious diseases among humans and animals, but they are widely used in the food industry, to increase meat production. ${ }^{9,10}$ A high proportion of administered antibiotics is discharged into water and soil through wastewater treatment plants, animal manure, sewage sludge, and biosolids frequently used to irrigate and fertilize agricultural lands. ${ }^{10}$ The release of antimicrobial agents into the aquatic environment and soil creates the conditions for the development of antibiotic resistant bacteria and the environmental occurrence of antibiotic resistant genes. ${ }^{9}$ Some of the main causes of ABR are listed in Figure 2.

The present review focuses specifically on ABR threat in humans, to highlight the attitude towards ABR in clinical practice settings all over the world.

\section{$A B R$ in Clinical Practice}

The emergence of ABR was typically considered as a problem predominantly concerning hospitals and care

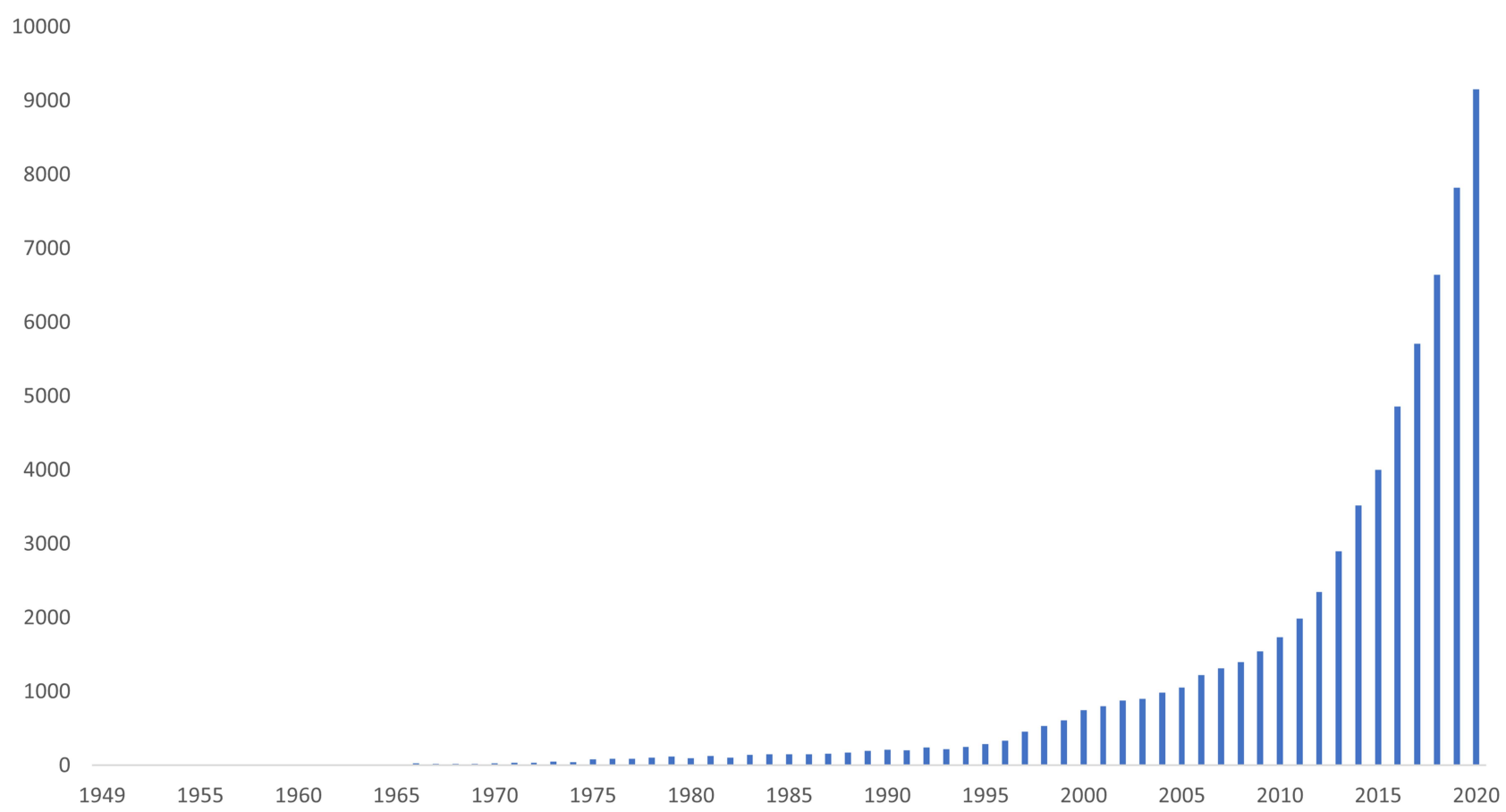

Figure I Timeline of publications indexed on PubMed related to ABR.

Note: Adapted from the PubMed timeline results (query: "antibiotic resistance" OR "antimicrobial resistance") by year. 


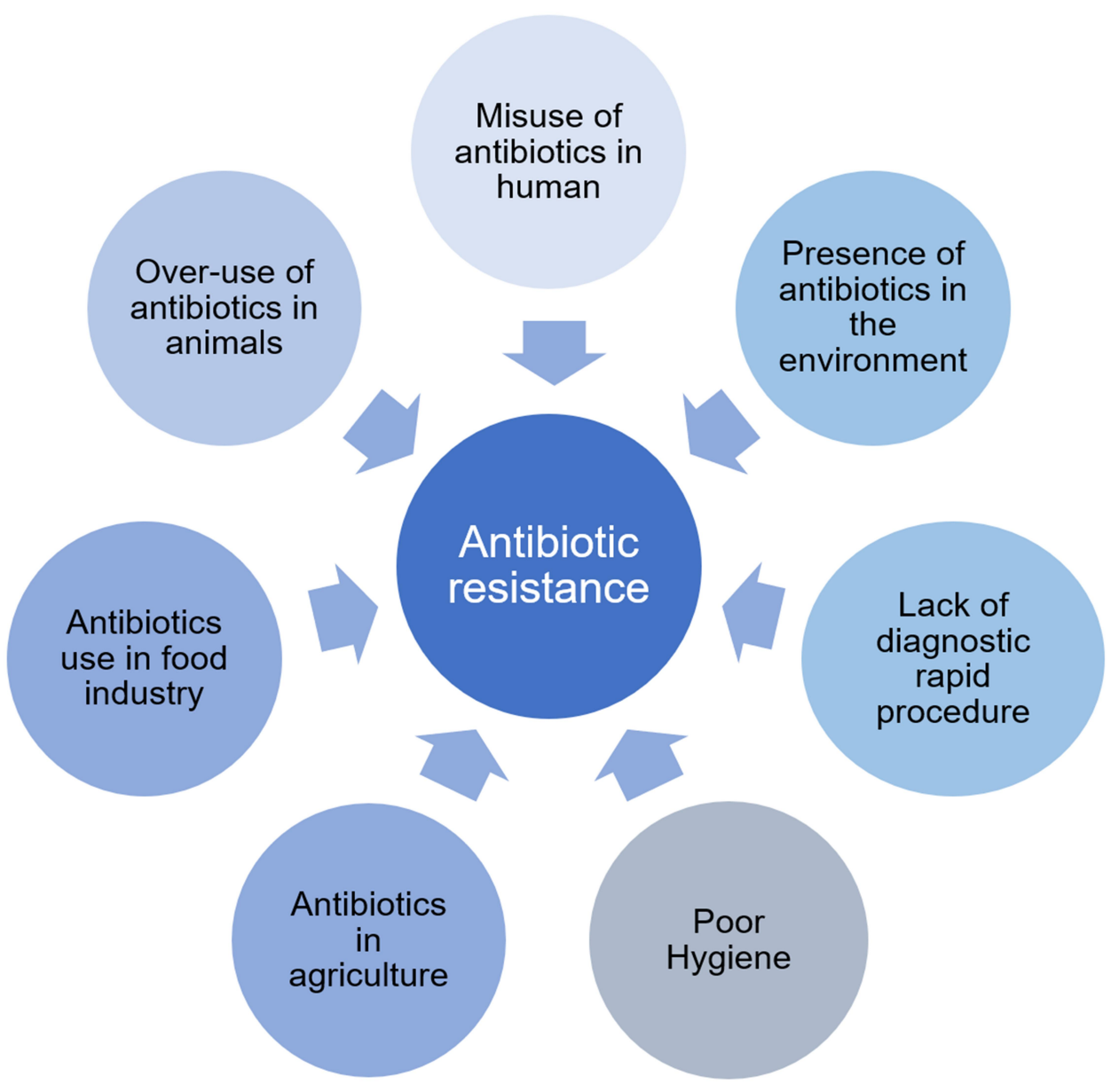

Figure 2 Main factors leading to antibiotic resistance.

facilities, however in the recent years resistant bacteria have been seen to spread in the wider community as well, leading to an increase in both the population at risk and the number of resistant infections. ${ }^{11,12}$ The misuse and abuse of antibiotics in clinical practice are among the main perpetrators for $\mathrm{ABR}$ and are now likely to undermine the incredible progress brought by antibiotics in modern medicine, including management of life-threatening infectious diseases or conditions such as cancer and advances made in surgical procedures, from caesarean sections to organ transplants. ${ }^{6}$

Infections are included among the major causes of death in the developing world and one of the responsible actors is the reappearance of once-controlled diseases due to ABR. ${ }^{13}$ Data on the epidemiology of infections caused by resistant bacteria are however scarce because of poor reporting and surveillance; in addition, the choice of the appropriate study design or statistical methods to calculate the incidence, risk of death or other clinical outcomes associated to infections with ABR bacteria is still open to debate. ${ }^{14-16}$ Cassini and co-workers recently provided an estimation of the burden of such infections based on data collected from the European Antimicrobial Resistance Surveillance Network (EARSNet) in 2015. ${ }^{14}$ Their findings showed that in the European Union and European Economic Area (EEA) countries, the number of cases of all types of infections (bloodstream, urinary tract, respiratory tract, surgical site and other infections) due to the selected ABR bacteria of public health importance was 671,689 (incidence of 131 infections per 100,000 population), $63.5 \%$ of which associated with healthcare facilities (hospitals and other health-care settings). Deaths attributable to these infections were estimated to be 33,110 (incidence of 6.44 deaths per 100,000 population), with approximately 11,000 reported only in Italy. Moreover, the authors for the first time reported the burden of infections for ABR expressed in disability-adjusted life-years (DALYs), that were found to be 874,541 , with overall DALY of 170 per 100,000 population, similar to the 
combined burden of HIV, influenza, and tuberculosis in 2015. The final "Review of antimicrobial resistance" chaired by $\mathrm{O}^{\prime} \mathrm{Neill}^{6}$ warned that, according to their estimation, $\mathrm{ABR}$ could cause approximately 10 million deaths yearly by 2050 unless global action is taken.

The picture described above is also exacerbated by the economic burden of ABR: a recent systematic review reported that health-care system costs were up to \$1 billion per year and estimated over \$3 trillion in Gross Domestic Product (GDP) loss. ${ }^{17}$

The aim of this review is to describe from a clinical practice standpoint the inappropriate use of antibiotics in terms of over-use, self-prescription, improper dosage regimens and treatment duration and to summarise the avoidable clinical outcomes and economic burden evaluated in observational studies (Figure 3).

\section{Development of ABR in Clinical Practice: The Role of Inappropriate Use of Antibiotics}

The inappropriate use of antibiotics is regarded as one of the main contributors to ABR onset. The misuse refers to high consumption of antibiotics, often unnecessarily, and it comprises over-use, inappropriate prescribing, self-medication, negligent use or incorrect dosage or treatment duration. ${ }^{18-20}$ Antibiotics misuse can be a consequence of patients' attitude, ${ }^{21}$ as evidenced by the high prevalence of use of antibiotics without prescription or by patients arbitrarily not following doctors' instructions nor the indication on the duration and dosage of treatments reported in the summary of product characteristics. ${ }^{22-24}$ Community pharmacists play a role too, in ensuring a cautious use of antibiotics. ${ }^{25}$

On the other hand, from a physicians' point of view, efforts should be made to optimize prescription patterns to avoid inappropriate antibiotic use - especially in primary health-care - in terms of over-prescription. ${ }^{26,27}$ In this regard, therapeutic inertia ${ }^{28}$ may play a role in using antibiotics for prolonged times when there are concerns about the outcome, uncertainty of diagnosis or in case of appearance of nonspecific symptoms. ${ }^{29,30}$

The adoption of guidelines/recommendations to guide the empirical choice of antibiotic therapy is pivotal. To this aim, Elias et $\mathrm{al}^{31}$ reviewed international guidelines of five frequent infectious syndromes (community-acquired pneumonia, urinary tract infections, acute otitis media, rhinosinusitis and pharyngitis) to evaluate the role of resistance patterns in the clinical decision-making. Disappointingly, the authors found in around two-thirds of the guidelines empiric recommendations not supported by data on resistance patterns, and very few guidelines reporting countryspecific resistance patterns. Similar conclusions could be drawn for other medical areas as well: for instance, in the

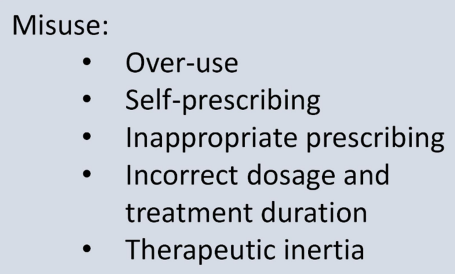

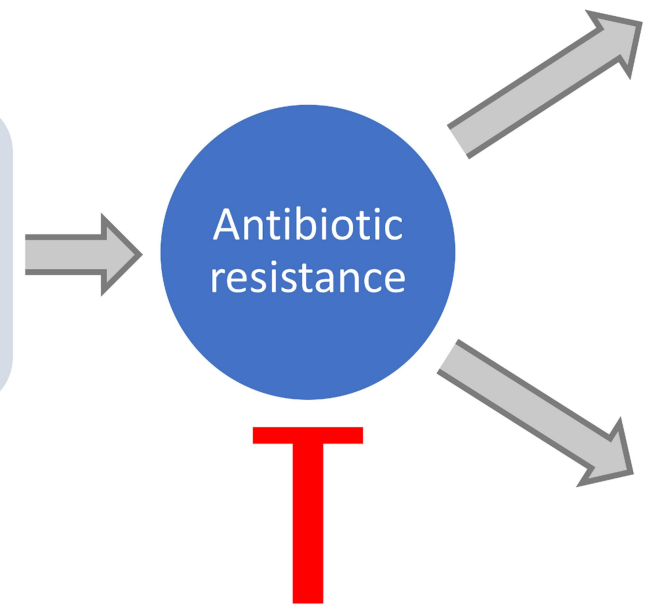

- Antibiotic stewardship
- Development of novel treatments
- Preventive measures
- Vaccines
- Rapid diagnostic techniques
Clinical burden:

Increased mortality

Increased morbidity

Longer hospitalization stays
Economic burden:

Cost of treatments

Cost of hospitalization

Loss of productivity

Medical services

Figure 3 Concept scheme: causes and consequences of antibiotic resistance in clinical practice. 
ophthalmology field, the standard of care is regarded from Miller $^{32}$ as "to treat first and culture later", and Grosso et $\mathrm{al}^{33}$ solicit that the use of antibiotics in ophthalmic surgery should be driven also by local microorganism surveillance. Moreover, Steels and van $\mathrm{Staa}^{34}$ observed that there is a need for bringing more clinically relevant evidence into guidelines to improve their uptake in clinical practice.

Several real-world studies performed in different countries highlighted the issue of antibiotic misuse and are reported in the following.

Considering studies based on the wide community, Smieszek et $\mathrm{al}^{35}$ performed a retrospective analysis based on the Health Improvement Network (THIN) database from 2013 to 2015 with the aim to quantify the inappropriate prescribing in primary care in England. Specifically, the authors focused on the over-prescription phenomenon, defined as "any antibiotic prescribing that is likely to have marginal or zero patient benefit and be outweighed by the potential risks of prescribing". To discriminate between appropriate and inappropriate prescribing, the authors took into account the treatment guidelines, the expert opinion of "ideal" prescribing proportions for defined conditions, and the variation in prescribing to identify overprescription. Three scenarios were presented: the most conservative considered the "most generous" estimates of appropriateness as per expert opinion; the middle scenario the narrowest over-prescribing definition; the least conservative scenario the "strictest" assumptions of the expert opinion. An inappropriate use of antibiotics was identified in all the scenarios applied, and accounted for $8.8 \%$ (most conservative), $15.4 \%$ (middle) and $23.1 \%$ (least conservative) of the prescriptions analysed. Furthermore, in all included practices there was a proportion of inappropriate prescriptions observed, which ranged from $3.6 \%$ to $52.9 \%$ (minimum of most conservative and maximum of least conservative scenarios, respectively).

The over-use of antibiotics is a well-documented problem in hospitals and in health-care facilities. Ciofi degli Atti et $\mathrm{al}^{36}$ conducted annual point prevalence surveys of hospital antibiotic consumption over the period 2008-2016 in the largest children's hospital in Italy: the prevalence of antibiotic use increased from $42.0 \%$ (2008) to $56.2 \%$ (2016). A similar trend (from $6.1 \%$ in 2008 to $24.2 \%$ in 2016) was also observed for the main indication, ie, medical prophylaxis, even though an improvement in the empirical choice of antibiotics was observed. As for misuse in primary care, acute respiratory tract infection is one of the health conditions most strongly related to antibiotic overuse. $^{37}$ Bianco et $\mathrm{al}^{27}$ described the antibiotic prescription pattern for acute respiratory tract infections in a southern Italian setting; results from this study showed that around two-thirds of patients were treated with antibiotics not indicated by guidelines. Consistent with the trend of antibiotic misuse in case of viral or mild bacterial infections, in a large nationwide US managed care network, Shekhawat et $\mathrm{al}^{38}$ reported approximately $60 \%$ of patients diagnosed with acute conjunctivitis received antibiotics.

The problem of self-medication is usually investigated by community surveys that allow for valuable information on antibiotic consumption to be collected, especially for low-income countries. ${ }^{39,40}$

Haddadin et al ${ }^{18}$ conducted a cross-sectional observational study based on a pool of pharmacies in Jordan to investigate the patterns of dispensed antibiotics in terms of appropriateness of drug indications, dosage and duration of treatments over a period of four months from February 2016 to May 2016. The results showed that around one-third of dispensation were without prescription and based on pharmacists' recommendation or on patients' request, and the authors speculated that this could be a consequence of the high proportion of people not covered by health-insurance. The $19 \%$ of non-prescribed antibiotics were given to patients diagnosed flu or cold, ie, to treat viral infections. Among the prescribed antibiotics dispensed, only $31.5 \%$ respected both the correct dosage and treatment duration according to the reference adopted. $^{41}$ These data were in line with another crosssectional study ${ }^{42}$ based on a survey submitted to Kuwaiti subjects during the period from January to March 2014, in which $27.5 \%$ of the study population have been taking antibiotics without medical consultation and with a recent community-based cross-sectional study conducted in Kemissie town (Ethiopia), in which one-quarter of the respondents did not obtain antibiotics with a prescription. ${ }^{43}$ Also a cross-sectional Italian analysis performed in $2011^{44}$ reported that around one-third of the respondents to the survey had taken an antibiotic without the prescription of a physician. A recent scoping review highlighted the selfmedication of antibiotics to be highly prevalent among individuals living in low- and middle-income countries. ${ }^{45}$ Such medications are often connected with improper use as fever, upper respiratory tract infection, common cold and sore throat. Information about antibiotics were mainly provided by pharmacists or a family member. ${ }^{45}$ 
The duration of antibiotic treatments also plays a crucial role in the development of ABR. While traditionally patients were recommended to complete a full antibiotic course to avoid ABR, a shorter course could be sufficient to treat bacterial infections in outpatient settings, and could represent a reliable strategy to reduce antibiotic consumption and to contrast the increasing rate of $\mathrm{ABR}^{46,47}$ In this direction were indeed the evidences described by Dawson-Hahn et $\mathrm{al}^{48}$ in an overview of systemic reviews of randomized controlled trials, that compared the effectiveness of short-courses to longcourses of oral antibiotics. Their findings indicated that for adults and children diagnosed with most common infections (tonsillopharyngitis, acute otitis media, uncomplicated urinary tract infection or mild/moderate community acquired pneumonia) managed in outpatient settings, short course antibiotics were as effective as longer course. The duration of antibiotic therapies as a threat for ABR was also under debate in the context of healthcare associated infections, specifically among the WHO recommendation for the prevention of surgical site infections. ${ }^{49}$ Even though guidelines recommend a postoperative duration of 24 hours at maximum, the expert panel advises against such prolongation of surgical antibiotic prophylaxis. This recommendation is based on a metanalysis conducted on 69 RCTs, in which prolonged regimens did not significantly reduce the incidence of surgical site infections compared with a single dose of antibiotic prophylaxis. ${ }^{49}$ Moreover, to avoid surgical site infections, the administration of prophylactic antibiotics therapy should cover the most common pathogens of the surgical site (that can be gram-positive or gram-negative bacteria), and should be administered for the short effective period, at a time ensuring an adequate serum concentration. ${ }^{50}$

High levels of antibiotic consumption are commonly encountered among residents of long-term care facilities. ${ }^{51}$ A population-based study performed in these institutions reported that around half of antibiotics administered had long treatment courses that exceed the "threshold" of one week. ${ }^{52}$ Notably, the use of prolonged therapies interested all antibiotic classes, including those for which a shorter duration was evidence-supported.

Administration of antibiotics for the shortest possible duration is one of the simplification regimen principles included in the concept of "antibiotic de-escalation". The latter is gaining widespread recognition as a key strategy for ABR stewardship. ${ }^{53,54}$ Briefly, de-escalation consists of modification of the initial broad-spectrum antibiotic therapy with a narrow-spectrum once the culture is available, and discontinuation of antimicrobial treatment if no infection is established. ${ }^{55}$ Moreover, de-escalation comprises reduction of the number of antibiotics and optimization of the antibiotic dose and route of administration. ${ }^{55}$ Similarly, in the choice of the appropriate empirical therapy for patients at high-risk of surgical site infection, a step-by-step reduction method is applied by administering broad-spectrum antibiotics at first, and adjusting the treatment according to the patient's response and the results of bacterial identification. ${ }^{56}$

\section{ABR Negative Outcomes}

The association of ABR with adverse health and economic outcomes is widely documented in literature. ${ }^{57-60} \mathrm{ABR}$ leads to long-lasting infections and causes a delay in the administration of microbiologically effective therapy. Moreover, due to the limited treatment options, patients affected with these infections may need toxic therapies administration, have prolonged hospitalizations or require surgical procedures. Overall, the impact of ABR results in increased morbidity and higher mortality rate, as well as in an increased resource utilization and higher costs. ${ }^{58}$

\section{Clinical Impact}

To better describe the population with infections caused by ABR organism and their negative clinical impact, observational studies focusing on different types of infection are presented.

Ryan et $\mathrm{al}^{61}$ conducted a retrospective study with the aim to investigate the prevalence of ABR bacteria affected by urosepsis and admitted in the emergency department of an Irish hospital between 2016 and 2018. Almost all patients included (97\%) reported a gramnegative organism as the causative pathogen. In the study population, a high level of ABR towards commonly prescribed antibiotics and broader agents (22\% piperacillin-tazobactam, $18 \%$ ciprofloxacin) were observed, underlying the actual challenge of choosing the appropriate therapy for these conditions. Patients from long-term care facilities were two-fold likely to be infected by an ABR organism compared to the community. Furthermore, patients with ABR were characterized as having more comorbidities such as diabetes, dementia, hypertension and ischaemic heart disease. Due to the relatively small sample size, no statistically significant difference in mortality or in length of hospitalization stays were detected between the resistant and non-resistant cohorts. On the 
contrary, Huang et $\mathrm{al}^{62}$ reported in their retrospective analysis that patients with urosepsis caused by extendedspectrum $\beta$-lactamase (ESBL) had a worse prognosis than the ones with non-ESBL urosepsis: the authors found a median length of hospitalization and of treatment duration of 4 days and 1 day longer, respectively. Furthermore, patients with ESBL urosepsis received less frequently the adequate antimicrobial therapy within 24 hours $(82.8 \%$ ESBL urosepsis vs $94.9 \%$ non-ESBL urosepsis, p-value 0.012). Ultimately, resistant patients experienced more frequently all-cause mortality or discharge with palliative measures.

Multidrug-resistant Acinetobacter baumannii (MRAB) strains have emerged over the past decades as one of the main causes of healthcare associated infections, particularly in intensive care unit. ${ }^{63,64}$ This pathogen is difficult to treat, and it is associated with high morbidity and mortality. ${ }^{63}$ Munier et $\mathrm{al}^{65}$ reported a prospective study aimed to evaluate the outcomes of MR-AB infection in a Burn Unit (BU) in France during an outbreak that occurred in 2014. MR-AB infections correlated with longer hospitalization stays, regardless of the outcome death/survival: considering only patients discharged alive, median length of hospital stay was 67 days in those MR-AB infected versus 19 days in patients not infected. The evaluation of the cause-specific hazard ratio (CSHR) revealed that MR-AB was associated with an increased risk of death (CSHR: 7.11; 95\% CI: 1.52-33.2; p-value 0.013 ). $57 \%$ of the deaths registered among patients with MR-AB was related to such infection, $29 \%$ were uncertainly related and $14 \%$ were unrelated.

ABR represents a serious issue for all those patients that require continuous hospitalization or that are repeatedly exposed to antibiotics, as in the case of liver cirrhosis. Bartoletti et al ${ }^{66}$ performed a large prospective multicentre cohort study on patients with liver cirrhosis who developed bloodstream infections (BSI). One of the main findings of the study concerned the high proportion (31\%) of BSI caused by multidrug-resistant organisms (MDRO), and the presence of these pathogens was strongly associated with inadequate empirical therapy and delays (during the first 24 hours) in effective treatment. These two last factors were in turn associated with increased rates of mortality. In addition, antimicrobial exposure or presence of invasive procedures in the 30 days prior BSI onset were identified as possible risk factors for MDRO.

\section{Economic Burden}

Economic burden of ABR was extensively reviewed over the past years. ${ }^{17,67-69}$ From the studies published in the literature, it seems clear that to estimate the costs for ABR is not an easy task, as different methodologies need to be applied, according to the perspective being taken. ${ }^{17}$ Costs from the patient perspective refer to associated mortality and morbidity, longer hospitalizations and higher toxic effects of second line drugs; costs from the health-care perspective consider additional diagnostic tests for resistant infection and reduced patient turnover and decreased revenues due to longer hospital admission. ${ }^{17,70}$ Alongside these perspectives, a secondary societal burden must be contemplated, that refers to the implication of ABR for medical procedure, eg, prophylactics in surgical procedure and antibiotic cotreatments for immunocompromising treatments. ${ }^{17,70}$

With these premises, Shrestha et $\mathrm{al}^{70}$ estimated the direct and indirect costs (US \$) of ABR (selecting key pathogens) per different classes of antibiotics consumed, using data from a low-middle income (Thailand) and high income (US) country to reproduce the variation in the global burden of ABR. For each resistant infection, direct costs ranged from $\$ 9.8$ to $\$ 29.0$ million in Thailand and from $\$ 9.5$ to $\$ 113.8$ million in US, while indirect costs spanned from $\$ 5$ to $\$ 56$ million and from $\$ 97$ to \$2184 million in Thailand and US, respectively. The total economic cost of ABR due to drug resistance in the pathogens considered was $\$ 0.5$ billion in Thailand and $\$ 2.8$ billion in US.

Consistent with these data, the costs estimated in Europe due to ABR per year were estimated to be $€ 1.5$ billion ( $\$ 1.65$ billion in US currency) considering health-care costs and productivity losses. ${ }^{71}$ According to O'Neill, the cost of taking global action for ABR is up to $\$ 40$ billion for the next years, little if compared to the $\$ 100$ trillion estimated as costs of inaction. ${ }^{6}$

Finally, a systemic review that evaluated the economic impact of antimicrobial stewardship programs reported a cost-saving mainly related to reduction in antibiotic consumption and direct costs, thus highlighting a potential positive health economic impact that warrant future research to be conducted to get insights also regarding hospitalization length and re-admission rates. ${ }^{72}$

\section{Conclusion}

To date, several programmes are currently ongoing to tackle the multifaceted health threat of $\mathrm{ABR} .^{73,74}$ Alternative 
approaches for the treatment of infectious diseases as antibodies, probiotics, vaccine development, phage therapy or small-molecule adjuvants affecting immune cells are in development. 4

This review provided a picture of the magnitude of ABR issue in clinical practice settings reporting population-based analysis/survey. The studies herein mentioned highlighted the misuse of antibiotics as one of the main contributors to ABR growing phenomenon. The misuse of antibiotics is often reported in terms of high consumption, overprescription of antibiotics, inappropriate prescribing, or selfprescription. Several aspects still need to be addressed to stop ABR from spreading; first of all, further active plans should be put in place to optimize the use of antibiotics also in terms of duration of treatments, and to minimize the over-use and inappropriate use of antibiotics. In this direction, antimicrobial stewardship takes into account decisions concerning the dose and duration of the most appropriate antibiotics to ensure minimal impact on local resistance levels and ensuring their availability and efficacy for the future. In addition, the diagnostic stewardship, ie, the implementation of rapid diagnostic techniques in clinical microbiology laboratories to aid the choice of drug therapy, is another emerging facet of antimicrobial stewardship. ${ }^{75}$ Moreover, ABR was associated in different studies with an increased risk of longer hospitalization and mortality. The clinical and economic burdens of ABR are huge and call for antibiotic stewardship programs to be taken and for increasing global surveillance networks.

\section{Disclosure}

The authors report no conflicts of interest in this work.

\section{References}

1. Blair JMA, Webber MA, Baylay AJ, Ogbolu DO, Piddock LJV. Molecular mechanisms of antibiotic resistance. Nat Rev Microbiol. 2015;13(1):42-51. doi:10.1038/nrmicro3380

2. Li B, Webster TJ. Bacteria antibiotic resistance: new challenges and opportunities for implant-associated orthopedic infections. J Orthop Res. 2018;36(1):22-32. doi:10.1002/jor.23656

3. Giedraitienè A, Vitkauskiené A, Naginiené R, Pavilonis A. Antibiotic resistance mechanisms of clinically important bacteria. Medicina. 2011;47(3):19. doi:10.3390/medicina47030019

4. Gajdács M. The concept of an ideal antibiotic: implications for drug design. Molecules. 2019;24(5):892.

5. Monserrat-Martinez A, Gambin Y, Sierecki E. Thinking outside the bug: molecular targets and strategies to overcome antibiotic resistance. Int J Mol Sci. 2019;20(6).

6. chaired by Jim O'Neill. Review on antimicrobial resistance. Tackling drug-resistant infections globally: final report and recommendations [Internet]; 2019. Available from: https://amr-review.org/Publications. html. Accessed February 15, 2021

7. Gajdács M, Albericio F. Antibiotic resistance: from the bench to patients. Antibiotics. 2019;8(3):129.
8. WHO. WHO | Global action plan on AMR [Internet]; 2019. Available from: http://www.who.int/antimicrobial-resistance/global-action-plan /en/. Accessed February 15, 2021.

9. Cycoń M, Mrozik A, Piotrowska-Seget Z. Antibiotics in the soil environment - degradation and their impact on microbial activity and diversity. Front Microbiol. 2019;10.

10. Felis E, Kalka J, Sochacki A, et al. Antimicrobial pharmaceuticals in the aquatic environment - occurrence and environmental implications. Eur J Pharmacol. 2020;866:172813. doi:10.1016/j. ejphar.2019.172813

11. Mulvey MR, Simor AE. Antimicrobial resistance in hospitals: how concerned should we be? CMAJ. 2009;180(4):408-415. doi:10.1503/ cmaj.080239

12. van Duin D, Paterson D. Multidrug resistant bacteria in the community: trends and lessons learned. Infect Dis Clin North Am. 2016;30 (2):377-390. doi:10.1016/j.idc.2016.02.004

13. Kapoor G, Saigal S, Elongavan A. Action and resistance mechanisms of antibiotics: a guide for clinicians. $J$ Anaesthesiol Clin Pharmacol. 2017;33(3):300-305. doi:10.4103/joacp.JOACP_349_15

14. Cassini A, Högberg LD, Plachouras D, et al. Attributable deaths and disability-adjusted life-years caused by infections with antibiotic-resistant bacteria in the EU and the European Economic Area in 2015: a population-level modelling analysis. Lancet Infect Dis. 2019;19(1):56-66. doi:10.1016/S1473-3099(18)30605-4

15. de Kraker MEA, Stewardson AJ, Harbarth S. Will 10 million people die a year due to antimicrobial resistance by 2050? PLoS Med. 2016;13(11):e1002184. doi:10.1371/journal.pmed.1002184

16. Wernli D, Jørgensen PS, Harbarth S, et al. Antimicrobial resistance: the complex challenge of measurement to inform policy and the public. PLoS Med. 2017;14(8):e1002378. doi:10.1371/journal.pmed.1002378

17. Naylor NR, Atun R, Zhu N, et al. Estimating the burden of antimicrobial resistance: a systematic literature review. Antimicrob Resist Infect Control. 2018;7:58. doi:10.1186/s13756-018-0336-y

18. Haddadin RN, Alsous M, Wazaify M, Tahaineh L. Evaluation of antibiotic dispensing practice in community pharmacies in Jordan: a cross sectional study. PLoS One. 2019;14(4):e0216115. doi:10.1371/journal.pone.0216115

19. Ventola CL. The antibiotic resistance crisis. Pharm Therap. 2015;40 (4):277-283.

20. Rather IA, Kim B-C, Bajpai VK, Park Y-H. Self-medication and antibiotic resistance: crisis, current challenges, and prevention. Saudi J Biol Sci. 2017;24(4):808-812. doi:10.1016/j.sjbs.2017.01.004

21. Davey P, Pagliari C, Hayes A. The patient's role in the spread and control of bacterial resistance to antibiotics. Clin Microbiol Infect. 2002;8:43-68. doi:10.1046/j.1469-0691.8.s.2.6.x

22. Liu J. Tackling the global non-prescription use of antibiotics. Lancet Infect Dis. 2020;20(2):169-170. doi:10.1016/S1473-3099(19)30744-3

23. Wogayehu B, Adinew A, Asfaw M. Knowledge on dispensed medications and its determinants among patients attending outpatient pharmacy at Chencha Primary Level Hospital, Southwest Ethiopia. Integr Pharm Res Pract. 2020;9:161-173. doi:10.2147/IPRP.S274406

24. Simon B, Kazaura M. Prevalence and factors associated with parents self-medicating under-fives with antibiotics in Bagamoyo District Council, Tanzania: a cross-sectional study. Patient Prefer Adherence. 2020;14:1445-1453. doi:10.2147/PPA.S263517

25. Gajdács M, Paulik E, Szabó A. Knowledge, attitude and practice of community pharmacists regarding antibiotic use and infectious diseases: a cross-sectional survey in Hungary (KAPPhA-HU). Antibiotics. 2020;9(2):41. doi:10.3390/antibiotics9020041

26. Wang J, Wang P, Wang X, Zheng Y, Xiao Y. Use and prescription of antibiotics in primary health care settings in China. JAMA Intern Med. 2014;174(12):1914-1920.

27. Bianco A, Papadopoli R, Mascaro V, Pileggi C, Pavia M. Antibiotic prescriptions to adults with acute respiratory tract infections by Italian general practitioners. Infect Drug Resist. 2018;11:2199-2205. doi:10.2147/IDR.S170349 
28. Gurwitz JH. The physics of geriatric pharmacotherapy: overcoming therapeutic inertia and momentum. Am J Med. 2012;125(6):523-524. doi:10.1016/j.amjmed.2012.02.007

29. May L, Gudger G, Armstrong P, et al. Multisite exploration of clinical decision-making for antibiotic use by emergency medicine providers using quantitative and qualitative methods. Infect Control Hosp Epidemiol. 2014;35(9):1114-1125. doi:10.1086/ 677637

30. Redberg RF. The problem of polypharmacy for nursing home residents comment on "prolonged antibiotic treatment in long-term care: role of the prescriber". JAMA Intern Med. 2013;173(8):683.

31. Elias C, Moja L, Mertz D, Loeb M, Forte G, Magrini N. Guideline recommendations and antimicrobial resistance: the need for a change. BMJ Open. 2017;7:e16264.

32. Miller D. Update on the epidemiology and antibiotic resistance of ocular infections. Middle East Afr J Ophthalmol. 2017;24(1):30-42.

33. Grosso A, Scarpa G, Romano M, et al. Choosing wisely: antibiotic use in ophthalmic surgery. Retina Today. 2019;48-53.

34. Steels S, van Staa TP. The role of real-world data in the development of treatment guidelines: a case study on guideline developers' opinions about using observational data on antibiotic prescribing in primary care. BMC Health Serv Res. 2019;19(1):942.

35. Smieszek T, Pouwels KB, Dolk FCK, et al. Potential for reducing inappropriate antibiotic prescribing in English primary care. J Antimicrob Chemother. 2018;73(suppl_2):ii36-43.

36. Ciofi Degli Atti ML, D'Amore C, Ceradini J, et al. Prevalence of antibiotic use in a tertiary care hospital in Italy, 2008-2016. Ital J Pediatr. 2019;45(1):63.

37. Löffler C, Krüger A, Daubmann A, et al. Optimizing antibiotic prescribing for acute respiratory tract infection in German primary care: study protocol for evaluation of the RESIST program. JMIR Res Protoc. 2020;9(9):e18648.

38. Shekhawat NS, Shtein RM, Blachley TS, Stein JD. Antibiotic prescription fills for acute conjunctivitis among enrollees in a large united states managed care network. Ophthalmology. 2017;124 (8):1099-1107.

39. Padget M, Guillemot D, Delarocque-Astagneau E. Measuring antibiotic consumption in low-income countries: a systematic review and integrative approach. Int $J$ Antimicrob Agents. 2016;48 (1):27-32.

40. Aslam A, Gajdács M, Zin CS, Binti Abd Rahman NS, Ahmed SI, Jamshed SQ. Public awareness and practices towards self-medication with antibiotics among the Malaysian population. A development of questionnaire and pilot-testing. Antibiotics. 2020;9(2):97.

41. Lexi-Comp I. Lexicomp Drug Information Handbook. Wolters Kluwer: American Pharmaceutical Association; 2019.

42. Awad AI, Aboud EA. Knowledge, attitude and practice towards antibiotic use among the public in Kuwait. PLoS One. 2015;10(2) e0117910.

43. Mengesha Y, Manaye B, Moges G. Assessment of public awareness, attitude, and practice regarding antibiotic resistance in Kemissie Town, Northeast Ethiopia: community-based cross-sectional study. Infect Drug Resist. 2020;13:3783-3789.

44. Napolitano F, Izzo MT, Giuseppe GD, Angelillo IF. Public knowledge, attitudes, and experience regarding the use of antibiotics in Italy. PLoS One. 2013;8(12):e84177.

45. Aslam A, Gajdács M, Zin CS, et al. Evidence of the practice of self-medication with antibiotics among the lay public in low- and middle-income countries: a scoping review. Antibiotics. 2020;9 (9):597.

46. Wald-Dickler N, Spellberg B. Short-course antibiotic therapy-replacing constantine units with "shorter is better". Clin Infect Dis. 2019;69(9):1476-1479.

47. Wilson HL, Daveson K, Del Mar CB. Optimal antimicrobial duration for common bacterial infections. Aust Prescr. 2019;42(1):5-9.
48. Dawson-Hahn EE, Mickan S, Onakpoya I, et al. Short-course versus long-course oral antibiotic treatment for infections treated in outpatient settings: a review of systematic reviews. Fam Pract. 2017;34(5):511-519.

49. Allegranzi B, Zayed B, Bischoff P, et al. New WHO recommendations on intraoperative and postoperative measures for surgical site infection prevention: an evidence-based global perspective. Lancet Infect Dis. 2016;16(12):e288-303.

50. Bratzler DW, Dellinger EP, Olsen KM, et al. Clinical practice guidelines for antimicrobial prophylaxis in surgery. Am J Health Syst Pharm. 2013;70(3):195-283.

51. Daneman N, Gruneir A, Newman A, et al. Antibiotic use in long-term care facilities. J Antimicrob Chemother. 2011;66 (12):2856-2863.

52. Daneman N, Gruneir A, Bronskill SE, et al. Prolonged antibiotic treatment in long-term care: role of the prescriber. JAMA Intern Med. 2013;173(8):673.

53. Daniel Markley J, Bernard S, Bearman G, Stevens MP. De-escalating antibiotic use in the inpatient setting: strategies, controversies, and challenges. Curr Infect Dis Rep. 2017;19(4):17.

54. Tabah A, Bassetti M, Kollef MH, et al. Antimicrobial de-escalation in critically ill patients: a position statement from a task force of the European Society of Intensive Care Medicine (ESICM) and European Society of Clinical Microbiology and Infectious Diseases (ESCMID) Critically Ill Patients Study Group (ESGCIP). Intensive Care Med. 2020;46(2):245-265.

55. Masterton RG. Antibiotic De-Escalation. Crit Care Clin. 2011;27 (1):149-162.

56. Han H-S, Park DJ. Antibiotic prophylaxis and surgical site infection prevention. In: Ljungqvist $\mathrm{O}$, Francis NK, Urman RD, editors. Enhanced Recovery After Surgery: A Complete Guide to Optimizing Outcomes [Internet]. Cham: Springer International Publishing; 2020:259-267.

57. Eliopoulos GM, Cosgrove SE, Carmeli Y. The impact of antimicrobial resistance on health and economic outcomes. Clin Infect Dis. 2003;36(11):1433-1437.

58. Friedman ND, Temkin E, Carmeli Y. The negative impact of antibiotic resistance. Clin Microbiol Infect. 2016;22(5):416-422.

59. Atif M, Asghar S, Mushtaq I, et al. What drives inappropriate use of antibiotics? A mixed methods study from Bahawalpur, Pakistan. IDR. 2019;12:687-699.

60. Vardakas KZ, Rafailidis PI, Konstantelias AA, Falagas ME. Predictors of mortality in patients with infections due to multi-drug resistant Gram negative bacteria: the study, the patient, the bug or the drug? J Infect. 2013;66(5):401-414.

61. Ryan J, McLornan L, O'Neill E. The impact of increasing antimicrobial resistance in the treatment of urosepsis. Ir J Med Sci. 2019.

62. Huang Y-WY, Alleyne A, Leung V, Chapman M. Urosepsis due to extended-spectrum $\beta$-lactamase-producing Escherichia coli: a retrospective, single-centre review of risk factors and clinical outcomes. Can J Hosp Pharm. 2018;71(2):119-127.

63. Silva ACB, Anchieta LM, Lopes de P, Romanelli de C. Inadequate use of antibiotics and increase in neonatal sepsis caused by resistant bacteria related to health care assistance: a systematic review. Braz J Infect Dis. 2018;22(4):328-337.

64. Peleg AY, Seifert H, Paterson DL. Acinetobacter baumannii: emergence of a successful pathogen. Clin Microbiol Rev. 2008;21 (3):538-582.

65. Munier A-L, Biard L, Legrand M, et al. Incidence, risk factors and outcome of multi-drug resistant Acinetobacter baumannii nosocomial infections during an outbreak in a burn unit. Int $J$ Infect Dis. 2019;79:179-184.

66. Bartoletti M, Giannella M, Lewis R, et al. A prospective multicentre study of the epidemiology and outcomes of bloodstream infection in cirrhotic patients. Clin Microbiol Infect. 2018;24(5):546.e1-546.e8. 
67. Gandra S, Barter DM, Laxminarayan R. Economic burden of antibiotic resistance: how much do we really know? Clin Microbiol Infect. 2014;20(10):973-980.

68. Tillotson GS, Zinner SH. Burden of antimicrobial resistance in an era of decreasing susceptibility. Expert Rev Anti Infect Ther. 2017;15 (7):663-676.

69. Leal JR, Conly J, Henderson EA, Manns BJ. How externalities impact an evaluation of strategies to prevent antimicrobial resistance in health care organizations. Antimicrob Resist Infect Control. 2017;6(1):53.

70. Shrestha P, Cooper BS, Coast J, et al. Enumerating the economic cost of antimicrobial resistance per antibiotic consumed to inform the evaluation of interventions affecting their use. Antimicrob Resist Infect Control. 2018;7:98.

71. Anderson M, Clift C, Schulze K, et al. Averting the AMR crisis: what are the avenues for policy action for countries in Europe? [Internet]. European observatory on health systems and policies; 2019. Available from: https://www.ncbi.nlm.nih.gov/books/NBK543412/. Accessed February 15, 2021.
72. Huebner C, Flessa S, Huebner N-O. The economic impact of antimicrobial stewardship programmes in hospitals: a systematic literature review. J Hosp Infect. 2019;102(4):369-376.

73. Birgand G, Castro-Sánchez E, Hansen S, et al. Comparison of governance approaches for the control of antimicrobial resistance: analysis of three European countries. Antimicrob Resist Infect Control. 2018;7(1):28.

74. Singh PK. One Health approach to tackle antimicrobial resistance in South East Asia. BMJ. 2017;358:j3625.

75. Morgan DJ, Malani P, Diekema DJ. Diagnostic stewardship-leveraging the laboratory to improve antimicrobial use. JAMA. 2017;318 (7):607-608.

\section{Publish your work in this journal}

Infection and Drug Resistance is an international, peer-reviewed openaccess journal that focuses on the optimal treatment of infection (bacterial, fungal and viral) and the development and institution of preventive strategies to minimize the development and spread of resistance. The journal is specifically concerned with the epidemiology of antibiotic resistance and the mechanisms of resistance development and diffusion in both hospitals and the community. The manuscript management system is completely online and includes a very quick and fair peerreview system, which is all easy to use. Visit http://www.dovepress.com/ testimonials.php to read real quotes from published authors. 